\title{
Spectra of black-hole binaries in the low/hard state: From radio to $X$-rays
}

\author{
D. Giannios
}

\author{
University of Crete, Physics Department, PO Box 2208, 710 03, Heraklion, Crete, Greece \\ e-mail: giannios@mpa-garching.mpg.de \\ Foundation for Research and Technology-Hellas, 711 10, Heraklion, Crete, Greece
}

Received 18 June 2004 / Accepted 21 March 2005

\begin{abstract}
We propose a jet model for the low/hard state of Galactic black-hole X-ray sources that can explain the energy spectra from radio to X-rays. The model assumes that i) there is a magnetic field along the axis of the jet; ii) the electron density in the jet drops inversely proportional to distance; and iii) the electrons in the jet follow a power law distribution function. We have performed Monte Carlo simulations of Compton upscattering of soft photons from the accretion disk and have found power-law high-energy spectra with photon-number index in the range 1.5-2 and cutoff at a few hundred keV. The spectrum at long wavelengths (radio, infrared, optical) is modeled to come from synchrotron radiation of the energetic electrons in the jet. We find flat to inverted radio spectra that extend from the radio up to about the optical band. For magnetic field strengths of the order of $10^{5}-10^{6} \mathrm{G}$ at the base of the jet, the calculated spectra agree well in slope and flux with the observations. Our model has the advantage over other existing models that it also explains many of the existing timing data such as the time lag spectra, the hardening of the power density spectra and the narrowing of the autocorrelation function with increasing photon energy.
\end{abstract}

Key words. accretion, accretion disks - black hole physics - radiation mechanisms: non-thermal methods: statistical - X-rays: stars

\section{Introduction}

Galactic black-hole binaries are classified by their X-ray features such as the strength and temperature of the soft multi color black-body component (Mitsuda et al. 1984), the hard $\mathrm{X}$-ray emission, the $\mathrm{X}$-ray luminosity and the timing properties. Several states have been identified to characterize black-hole accretion (for reviews see Tanaka \& Lewin 1995; van der Klis 1995; Nowak 1995; Poutanen 1998; McClintock \& Remillard 2005). A source is in the so-called low/hard state when it exhibits low X-ray luminosity (less than a few \% the Eddington luminosity $L_{\mathrm{E}}$ ), weak or absent thermal component, dominant hard X-ray emission with a cutoff at $\sim$ a few hundred $\mathrm{keV}$ and high rms variability.

The soft thermal component is widely accepted to come from an optically thick, geometrically thin accretion disk (Shakura \& Sunyaev 1973), while the hard X-ray tail is usually assumed to come from inverse Compton scattering of soft photons off energetic electrons (e.g. Sunyaev \& Titarchuk 1980). The location of the energetic electrons and, more generally, the accretion geometry are poorly understood. One possibility is that the disk extends all the way down to the last stable circular orbit and that there is a hot rarefied "corona" that lies above and below the thin disk (Galeev et al. 1979; Haardt \& Maraschi 1993; Haardt et al. 1994; Stern et al. 1995; Poutanen \& Fabian 1999). It is also possible that the disk is truncated at a larger radius and the inner region is filled with optically thin, geometrically thick, two-temperature plasma (Shapiro et al. 1976; Ichimaru 1977; Rees et al. 1982; Narayan \& Yi 1994; Abramowicz et al. 1995; Esin et al. 1998).

During recent years, evidence has mounted that, when in the low/hard state, X-ray binaries exhibit steady core radio emission (Hjellming \& Han 1995; Mirabel et al. 1998; Fender 2001; Gallo et al. 2003). Moreover, in some cases a jet-like structure has been resolved (Mirabel et al. 1992; Dhawan et al. 2000; Stirling et al. 2001; Martí et al. 2002; Fuchs et al. 2003). The spectrum in the radio is flat to inverted and seems to extend to the infrared or even beyond (see, e.g., Hannikainen et al. 1998; Fender et al. 2000; Corbel et al. 2000). Because of the high brightness temperatures, non-thermal spectra and, in some cases, high degree of polarization, radio emission is believed to come from synchrotron radiation of relativistic electrons in a jet. Furthermore, an interesting radio/X-ray correlation has been established to hold through simultaneous radio and X-ray observations (Gallo et al. 2003) which can be extended to include the mass of the black hole and to define the "fundamental plane" of black hole activity (Merloni et al. 2003; see also Falcke et al. 2004).

Inverse Compton scattering by relativistic electrons in a jet has been proposed as a mechanism for the production of $\mathrm{X}$-rays and $\gamma$-rays in X-ray binaries (Band \& Grindlay 1986; Levinson \& Blandford 1996; Georganopoulos et al. 2002; 
Romero et al. 2002). The possibility that optically thin synchrotron emission from the jet results in the hard X-ray tail has also been explored (Markoff et al. 2001; Vadawale et al. 2001; Corbel \& Fender 2002; Markoff et al. 2003; see also Markoff $\&$ Nowak 2004, for constraints on jet models via reflection).

Recently, Reig et al. (2003, hereafter Paper I) proposed a jet model that can explain the X-ray energy spectra and the dependence of time lags on Fourier frequency (see for example Miyamoto et al. 1988; Nowak et al. 1999; Ford et al. 1999) in terms of inverse Compton scattering of soft, disk photons by energetic electrons in the jet. The density of the electrons in this simple jet model is assumed to drop inversely proportional to the vertical distance $z$ from the black hole. Giannios et al. (2004, hereafter Paper II; see also Kylafis et al. 2004) further assumed that the electrons close to the core of the jet are more energetic than those at its periphery and showed that both the hardening of the high-frequency power spectra and the narrowing of the auto-correlation function with photon energy observed in Cygnus X-1 (Nowak et al. 1999; Revnivtsev et al. 2000; Maccarone et al. 2000) are reproduced.

Although successful in explaining a large number of spectral and timing properties in the X-ray domain, Papers I, II did not deal with the part of the electromagnetic spectrum that revealed the existence of the jet in the first place, i.e. the radio. Radio observations on the other hand contain valuable information (spectral slope, flux) that can place constrains on any jet model. In this work, we model the radio emission in terms of synchrotron radiation of the electrons in the jet. The main modification with respect to the jet model used in Papers I, II is that we take the electrons to have a power law energy distribution function. We first make sure that this modification does not alter any of the previously derived results in the X-ray domain. This puts significant constraints on the index of the power law energy distribution of the electrons. Then we show that flat to inverted radio spectra are naturally reproduced by the model. Finally, we apply the model to the broad band spectra of XTE J1118+480 and Cygnus X-1.

\section{The model}

\subsection{Description of the jet}

The jet model that we will use here is built on the model of Paper I. Here we describe the characteristics of the jet. We assume that the jet is accelerated close to its launching region and that it has constant velocity $v_{\|}$(say along the $z$-axis). Furthermore, we assume that the electron density in the jet drops inversely proportional with distance $z$

$n(z)=n_{0} \frac{z_{0}}{z}$

where $n_{0}$ is the density and $z_{0}$ is the height at the base of the jet respectively. Mass conservation $\dot{M} \propto v_{\|} n(z) R^{2}(z)$ then determines the polar radius $R$ of the outer edge of the jet as a function of $z$

$R(z)=R_{0}\left(\frac{z}{z_{0}}\right)^{1 / 2}$, where $R_{0}$ is the radius at the base of the jet. The Thomson optical depth along the axis of the jet is given by the integration of $\mathrm{d} \tau=n(z) \sigma_{\mathrm{T}} \mathrm{d} z$ from $z_{0}$ to $H$ (where $H$ is the extent of the jet)

$\tau_{\|}=n_{0} \sigma_{\mathrm{T}} z_{0} \ln \left(\frac{H}{z_{0}}\right)$.

In the previous works (Papers I, II) the magnetic field had been assumed, for simplicity, to be parallel to the axis of the jet (z-axis) and homogeneous. Here, we will keep $\boldsymbol{B} \| z$ but take its strength to vary with $z$ as dictated by magnetic flux conservation along the jet: $B(z) \pi R^{2}(z)=$ const. So we have for the $z$-dependence of the magnetic field

$B(z)=B_{0} \frac{z_{0}}{z}$

The main modification with respect to Papers I and II is in the energy distribution of the electrons. In Paper I, the electrons had been taken monoenergetic with their velocities to have a constant spiraling perpendicular component $v_{\perp}$. In Paper II, $v_{\perp}$ had been assumed to drop linearly with polar distance by a factor of a few (resulting in a jet with a core "hotter" than its periphery), explaining the hardening of the high-frequency power spectrum with increasing photon energy in Cygnus X-1 (Nowak et al. 1999).

Here we will assume that the electrons have a distribution of $v_{\perp}$ that extends to ultra-relativistic electron velocities. We will describe the distribution in terms of the Lorentz factor

$\gamma=1 / \sqrt{1-\left(v_{\|}^{2}+v_{\perp}^{2}\right) / c^{2}}$

and will assume - as it is customary in many jet models (e.g. Blandford \& Königl 1979) - a power law form

$N(\gamma) \mathrm{d} \gamma \propto \gamma^{-\alpha} \mathrm{d} \gamma$.

The distribution extends from $\gamma_{\min }$ to $\gamma_{\max }$ with $\gamma_{\max } \gg \gamma_{\min }$. In total, three parameters are needed to determine the electron distribution inside the jet i.e. the index $\alpha, \gamma_{\min }$, and $\gamma_{\max }$. Since the distribution itself is expressed in terms of the Lorentz factor $\gamma$, we prefer to keep $\gamma_{\min }$ as a parameter instead of $v_{\perp}$ (of course these quantities are related through Eq. (5)).

With this model at hand, the task is to explore its spectral properties in essentially the whole electromagnetic spectrum so as to compare with those observed in black-hole candidates in the low/hard state. Synchrotron emission from the energetic electrons inside the magnetic field given by Eq. (4) will be shown to dominate from the radio up to about the optical wavelengths, while we model the hard X-ray part of the spectrum as a result of inverse Compton scattering of a soft-photon input at the base of the jet by the same population of electrons. We have used the Monte Carlo method (Pozdnyakov et al. 1983) to simulate inverse Compton scattering.

\subsection{Simulations of inverse Compton scattering in the jet}

Our code is very similar to the one described in Papers I and II. Here, we describe only the emission of the soft photons. Multi-color black-body photons (i.e. accretion disk photons) of 
maximum temperature $T_{\mathrm{bb}}$ are assumed to be emitted from the underlying disk with an upward isotropic distribution and temperature that scales with polar radius as $T \propto \rho^{-3 / 4}$ (Shakura \& Sunyaev 1973). Furthermore, synchrotron flux from the jet also contributes to the soft photon input to be Comptonized and is taken into account when the model is applied to observations (see Sect. 6).

\section{Model parameters}

The present model has several free parameters, most of which refer to physical quantities of the jet. These are: the temperature $T_{\mathrm{bb}}$, the extent $H$ of the jet, the radius $R_{0}$ and the height $z_{0}$ of the base of the jet, the Thomson optical depth $\tau_{\|}$along the axis of the jet, the velocity of the jet $v_{\|}$and the strength of the magnetic field at the base of the jet $B_{0}$. The electron distribution is specified through the minimum Lorentz factor $\gamma_{\min }$, the maximum Lorentz factor $\gamma_{\max }$ and the exponent of the electron distribution $\alpha$ (see Eqs. (5) and (6)).

The values of the parameters that reproduce quite well several properties of black-hole candidates in the low/hard state (with special emphasis to Cygnus X-1) are called reference values in this work and they are: $k T_{\mathrm{bb}}=0.2 \mathrm{keV}$, $H=5 \times 10^{8} r_{\mathrm{g}}, R_{0}=100 \mathrm{rg}, z_{0}=5 r_{\mathrm{g}}, \tau_{\|}=2.5, v_{\|}=0.8 c$, $B_{0}=3 \times 10^{5} \mathrm{G}, \gamma_{\min }=2.1, \gamma_{\max }=500$ and $\alpha=4$, where $r_{\mathrm{g}}=G M / c^{2} \simeq 1.5 \times 10^{6} \mathrm{~cm}$ corresponds to the gravitational radius of a 10 solar-mass black hole.

Since the jet is mildly relativistic, the results also depend on the angle $\theta$ of observation with respect to the jet axis. As in Paper II, we will focus in an intermediate range of observing angles $0.2<\cos \theta<0.6$. Practically, for the Monte Carlo simulation this means that we count only photons that leave the jet in this range of angles.

Despite the fact that the parameter space is rather large, all the parameters can be constrained since the model is tested against (and succeeds in reproducing) a large number of timing and spectral properties of black-hole X-ray binaries. It is encouraging that none of the parameters needs to be fine tuned in order to match the observations. The last statement will be quantified in the next sections, where the results are presented.

\subsection{The ejection rates}

Using the reference values, one can calculate the number density $n_{0}$ of the electrons (plus possibly positrons) at the base of the jet (Eq. (3)). Assuming a neutral jet and $f$ electrons per proton (which also means $f-1$ positrons), the ejection rate in the jet is given by the expression

$\dot{M}=n_{0} \pi R_{0}^{2} v_{\|}\left(m_{\mathrm{e}}+\frac{m_{\mathrm{p}}}{2 f-1}\right)$.

In the case of absence of pairs (i.e. $f=1$ ) and using the reference values of the parameters one finds an ejection rate $\dot{M}=8.2 \times 10^{19} \mathrm{~g} \mathrm{~s}^{-1}$. This value is super Eddington but not necessarily unrealistic. In the case, however, of a pair dominated jet (i.e. $f \gg 1$ ) the ejection rate can be lower by more than three orders of magnitude and much more "reasonable". At this point, our knowledge on the composition of the jet is poor and both baryon and pair dominated jets are allowed by observations (see for example Fender 2003).

\subsection{The spectral index of the electron distribution}

In this work we treat the power-law index of the electron distribution $\alpha$ as a free parameter and we do not address the issue of particle acceleration in the jet. However, the values of $\alpha \gtrsim 3$ (see next section) that we use are unusually large (with respect to what is expected from shock acceleration for example). Here, we show that $\alpha$ is not the spectral index of the injected electrons but that of the (steady-state) emitting electrons after taking into account radiative (synchrotron and inverse Compton) cooling.

Defining the "cooling Lorentz factor" $\gamma_{\mathrm{c}}$ of the electrons as the Lorentz factor at which the radiative cooling time scale $t_{\mathrm{rad}}$ equals the dynamical time scale $t_{\text {dyn }}$, where

$t_{\mathrm{dyn}}=\frac{z}{c}=t_{\mathrm{rad}}=\frac{t_{\mathrm{syn}} t_{\text {Compton }}}{t_{\mathrm{syn}}+t_{\text {Compton }}}$,

one can estimate $\gamma_{\mathrm{c}}$. The synchrotron cooling time $t_{\mathrm{syn}}$ can be calculated straightforwardly with the use of Eq. (4), while the Compton cooling time can be calculated numerically with the Monte Carlo method (see Sect. 2.2). For the reference values of the parameters of the jet it is estimated to be $\gamma_{\mathrm{c}} \simeq \mathrm{a}$ few $\simeq$ $\gamma_{\min }$. So, it is approximately correct to assume that the whole electron distribution is in the fast cooling regime and can be modeled as a power-law.

\section{Results in the X-ray domain}

In this section, we show our results concerning the spectral properties of the electromagnetic radiation in the X-rays. The temporal properties are discussed very briefly since a detailed description has already been given in Paper II.

\subsection{Energy spectra}

In Fig. 1 (solid curve) we plot the energy spectrum of the emerging radiation as calculated by our Monte Carlo simulation for the reference values of the parameters (see Sect. 3) and for a black-body soft photon input ${ }^{1}$. Two components are evident in this plot. Below $\sim 1 \mathrm{keV}$ the dominant component is the soft photon input, while for $E \gtrsim 1 \mathrm{keV}$ the Comptonized component dominates. The latter component can be well fitted with a power law of photon-number index $\Gamma \simeq 1.7$ with an exponential cutoff at $E_{\text {cut }} \simeq 300 \mathrm{keV}$.

In Papers I, II we have explored how the spectrum depends on some of the parameters such as the optical depth along the jet $\tau_{\|}$or the extent of the base of the jet $R_{0}$. The result was that increasing $\tau_{\|}$or $R_{0}$, while keeping the rest of the parameters fixed, the spectrum becomes harder but with essentially the

\footnotetext{
1 Since in this section we explore how the model parameters affect the X-ray spectra (and mainly the X-ray slope and high energy cutoff), the energy distribution of the soft photon input is not very important. In Sect. 6, however, where the model is applied to data, all relevant soft photon sources such as multi-temperature disk and synchrotron photons are taken into account.
} 


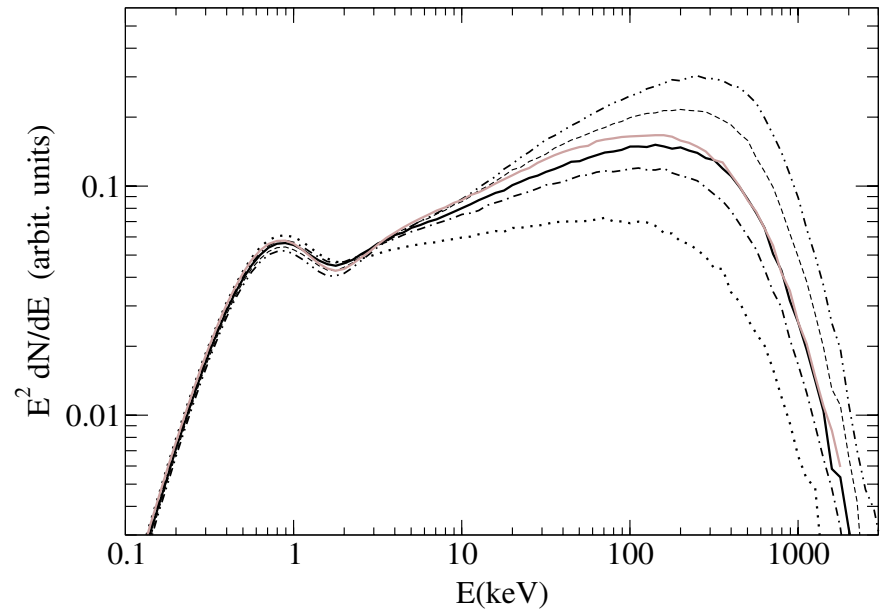

Fig. 1. Emergent photon-number spectrum multiplied by $E^{2}$ from our jet model for different values of the minimum Lorentz factor $\gamma_{\min }$ of the electron distribution. The solid curve corresponds to the reference values of the parameters. The dotted, dash-dotted, dashed and dashdouble-dotted curves correspond to $\gamma_{\min }=1.8,2,2.3$, and 2.5 respectively. The rest of the parameters are kept at their reference values. The solid gray curve corresponds to the illustrative example where $\gamma_{\min }$ varies with $z$ according to Eq. (9).

same energy cutoff. This is expected since in both cases there is an increase of the mean number of scatterings that the electrons experience during their random walk inside the jet and gain more energy on average. The cutoff, on the other hand, is mainly determined by the energetics of the electrons which does not depend on $\tau_{\|}$or $R_{0}$. The same conclusions have been checked to hold in this version of the model.

Of more interest is to explore how the energetics of the electrons influence the emerging spectrum. For a power-law distribution of electrons, both $\gamma_{\min }$ and the index $\alpha$ are relevant quantities for this exploration. For $\alpha$ large enough, as is the case here (see below), the exact value of $\gamma_{\max }$ is not important. It is qualitatively expected that by increasing $\gamma_{\min }$ or making the distribution flatter (i.e. decreasing $\alpha$ ), the spectrum becomes harder and the exponential cutoff appears at higher energies.

In Fig. 1 we plot the emerging spectrum for different values of $\gamma_{\min }$, while the rest of the parameters are kept at their reference values. One can clearly see the hardening of the spectrum with increasing $\gamma_{\min }$. More quantitatively, by increasing $\gamma_{\min }$ in the range $1.8-2.5$, the photon number index $\Gamma$ of the hard $\mathrm{X}$-ray slope hardens from $\sim 1.9$ to $\sim 1.5$ which is well within the observed range for black-hole binaries in the low/hard state. For simplicity, we have assumed that $\gamma_{\min }$ is constant along the jet. This, however, may not be the case and $\gamma_{\min }$ may vary as a function of $z$. We have checked that if $\gamma_{\min }$ changes rather slowly with $z$, the resulting spectra do not change much. As an example, in Fig. 1 we plot the X-ray spectrum for

$\gamma_{\min }=\gamma_{1}\left(1+0.8 \sqrt{z_{0} / z}\right)$

where $\gamma_{1}=1 / \sqrt{1-v_{\|}^{2} / c^{2}}$.

We also show (in Fig. 2) how the emergent spectrum depends on the index $\alpha$ of the electron distribution. As $\alpha$

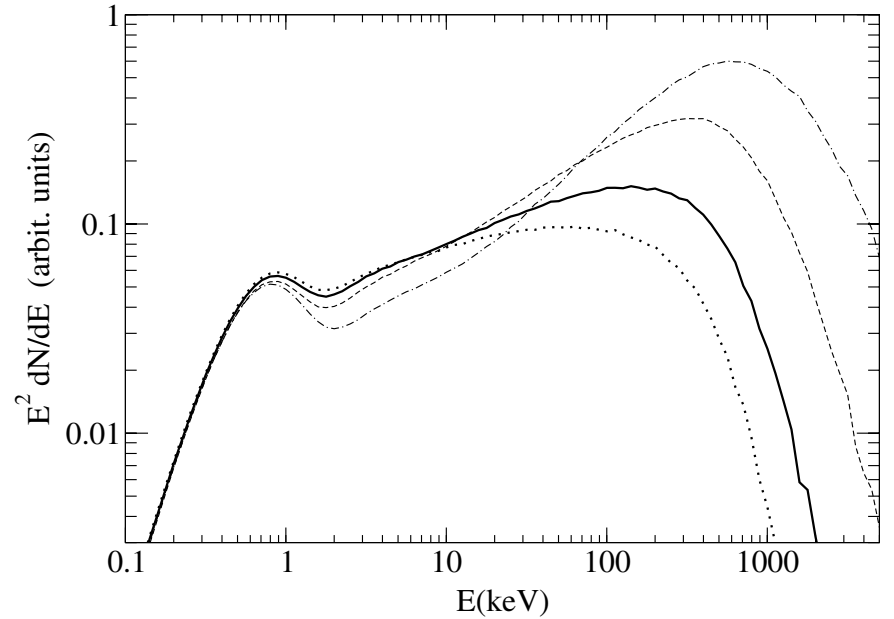

Fig. 2. Emergent photon-number spectrum multiplied by $E^{2}$ from our jet model for different values of the power-law index $\alpha$. The dotted, solid, and dashed curves correspond to $\alpha=5,4,3$ respectively. We also plot (dash-dotted curve) the case where $\alpha=2$ and $\gamma_{\min }=1.67$. The rest of the parameters are kept to their reference values.

decreases, the spectrum gets harder with a cutoff at higher energies. Notice that for $\alpha=2$ we use the minimum value one could possibly have for $\gamma_{\min }$, namely $\gamma_{\min }=1 / \sqrt{1-v_{\|}^{2} / c^{2}}=1.67$ (see Eq. (5)). One important conclusion that can be drawn from this figure is that if the electron distribution is rather flat (say $\alpha \sim 2$ ), the high energy cutoff becomes too high ( $E_{\text {cut }} \simeq 1 \mathrm{MeV}$ or more) in comparison with the one typically observed in black-hole binaries in the low/hard state $(\sim$ a few hundred $\mathrm{keV}$ ) for any choice of $\gamma_{\mathrm{min}}$. This consideration requires $\alpha \gtrsim 3$ if this model is to agree with spectral observations. As we have already discussed in Sect. 3.2., such a steep spectral index is the result of fast radiative (synchrotron and inverse Compton) cooling of the electrons.

\subsection{Temporal domain}

In Papers I and II, it has been shown that the jet model can account for a number of temporal properties of X-ray blackhole binaries. Since in this work we have modified the energetics of the electrons, it is important to check whether the previously derived results are altered or not. For example the Fourier frequency dependence of the time lags between two different energy bands is found to remain essentially the same as that shown in Papers I and II, in accordance with observations (see for example Nowak et al. 1999; Ford et al. 1999). Furthermore, the width of the autocorrelation function has been checked to decrease with photon energy (as pointed out observationally by Maccarone et al. 2000). Finally, it was shown in Paper II that, assuming that more energetic electrons lie in the core of the jet in comparison to its periphery, a hardening of the highfrequency power spectrum with increasing photon energy is expected. If we assume - in the context of the current model- that the minimum Lorentz factor $\gamma_{\text {min }}$ decreases with polar distance, a similar hardening of the power spectrum is derived. 


\section{Radio emission from the jet}

The existence of a steady jet when a black-hole binary is in the low/hard state has been strongly suggested through radio observations (Hjelling \& Han 1995; Mirabel \& Rodríguez 1999; Fender 2001). Furthermore, in some occasions a jet like structure has even been resolved (Mirabel et al. 1992; Dhawan et al. 2000; Stirling et al. 2001; Martí et al. 2002; Fuchs et al. 2003). Our simple jet model has succeeded in explaining a large number of spectral and timing properties of these sources in the $\mathrm{X}$-ray region in terms of inverse Compton scattering of soft photons by energetic electrons in the jet. Here we will focus on longer wavelengths and explore the predictions of our model in this region of the electromagnetic spectrum.

\subsection{The spectral slope in the radio}

The coexistence in the jet of energetic electrons and magnetic fields indicates synchrotron emission as the dominant radiative mechanism in the radio. The study of radio emission is more straightforward in a frame that is comoving with the emitting medium (i.e. the jet). Suppose that an electron has a Lorentz factor $\gamma$ in the lab frame (i.e. the rest frame of the black hole). Then a Lorentz transformation gives the Lorentz factor in the comoving frame

$\gamma_{\mathrm{co}}=\sqrt{1-v_{\|}^{2} / c^{2}} \gamma$.

Since we have assumed that $v_{\|}$is constant in the jet, $\gamma_{\mathrm{co}} \propto \gamma$. Stated in other words, we still have a power law distribution for the electrons in the comoving frame, namely

$N\left(\gamma_{\mathrm{co}}\right) \mathrm{d} \gamma_{\mathrm{co}}=C \gamma_{\mathrm{co}}^{-\alpha} \mathrm{d} \gamma_{\mathrm{co}}$.

For the rest of this work, and for simplicity in the notation, we will drop the subscript "co" from the Lorentz factor. The constant $C$ can be found if we integrate the last expression from $\gamma_{\min }$ to $\gamma_{\max } \gg \gamma_{\min }$ and equate it to the comoving electron density. Doing so we have

$C=n \sqrt{1-v_{\|}^{2} / c^{2}}(\alpha-1) \gamma_{\min }^{\alpha-1}$,

where we have also assumed that $\alpha>1$, which certainly holds, and $n$ is given by Eq. (1).

The properties of the jet (i.e. density, magnetic field strength, energetics of the electrons) may vary only along the axis of the jet but not along the perpendicular direction. As is customary, we can exploit this symmetry by dividing the jet into "slices" across the $z$-axis and calculating the synchrotron emission and absorption at each slice separately. Adding the contribution of all slices, we get the total emitted power.

For a relativistic power law distribution of electrons, the emitted synchrotron power per unit frequency $\mathrm{d} P(v)$ in a slice in the range $(z, z+\mathrm{d} z)$ is (see, e.g., Eq. (6.36) of Rybicki \& Lightman 1979)

$\mathrm{d} P(v)=C_{\alpha} B^{\frac{\alpha+1}{2}} \gamma_{\min }^{\alpha-1} n \sqrt{1-v_{\|}^{2} / c^{2}} v^{\frac{1-\alpha}{2}} R^{2} \mathrm{~d} z$,

where $C_{\alpha}$ depends only on the index $\alpha$ (defined in Eq. (6)), while $B=B(z), n=n(z)$, and $R=R(z)$ are given by Eqs. (4), (1), and (2), respectively.
The synchrotron radiation is strongly self-absorbed below a characteristic frequency (the turn-over frequency) $v_{\mathrm{t}}$. Synchrotron absorption coefficient is given by the expression (see Eq. (6.53) in Rybicki \& Lightman 1979)

$a_{v}=A_{\alpha} n \gamma_{\min }^{\alpha-1} B^{\frac{\alpha+2}{2}} v^{-\frac{\alpha+4}{2}}$,

where $A_{\alpha}$ depends only on the index $\alpha$. The turn-over frequency in a slice of the jet can be estimated as the frequency for which the optical depth to synchrotron absorption, across a radius of this slice becomes unity, i.e. $a_{v_{\mathrm{t}}} R(z) \simeq 1$. Solving this expression for $v_{\mathrm{t}}$ and using Eqs. (1), (2), (4), and (14) we have

$v_{\mathrm{t}}=A_{\alpha}^{-\frac{2}{\alpha+4}} n_{0}^{-\frac{2}{\alpha+4}} \gamma_{\min }^{\frac{2 \alpha-2}{\alpha+4}} B_{0}^{\frac{\alpha+2}{\alpha+4}}\left(\frac{z_{0}}{z}\right)^{\frac{\alpha+3}{\alpha+4}}$.

In estimating the turn-over frequency by setting $a_{v_{\mathrm{t}}} R(z) \simeq 1$, we actually assume that the jet thickness $R(z)$ is much less than its height $z$. This does not hold close to the base of the jet where $z<R(z)$. From Eq. (2) we can verify that $z \gg R(z)$ when $z \gg 10^{3} r_{\mathrm{g}}$. Since the jet extends up $H=5 \times 10^{8} r_{\mathrm{g}}$, our assumption holds along most of the jet. We will return to this discussion at the end of Sect. 5.2.

Before proceeding to a more detailed calculation of the emitted spectrum, we will calculate the slope of the spectrum over a large range of frequencies. It is clear from Eq. (15) how the turn-over frequency drops with the height $z$ of the slice of the jet. Since $z$ extends over many orders of magnitude from $z_{0}$ up to $H$, there is a large range of frequencies $v$ for which part of the jet is optically thin and the rest optically thick. The height $z_{v}$ of the transition from the optically thin region to the optically thick one depends on the frequency and is given by Eq. (15) if we solve for $z$ and call it $z_{v}$, namely

$z_{v} \propto v^{-\frac{\alpha+4}{\alpha+3}}$.

If, for the moment, we take into account only the optically thin part of synchrotron emission, to calculate the power emitted at frequency $v$, one has to integrate Eq. (13) from $z_{v}$ to $H$ and use Eq. (16) to arrive to the scaling

$P(v) \propto v^{\frac{\alpha-1}{2 \alpha+6}}$.

The slope $\frac{\alpha-1}{2 \alpha+6}$ depends rather weakly on $\alpha$ and for $3 \lesssim \alpha \lesssim 5$, the slope is $\sim 0.2$. This result, which will be verified by the more detailed calculation of Sect. 5.2, shows that the emitted spectra of our model agree very well with the flat to inverted radio spectra that are typically observed from the steady jets of black hole candidates in the low/hard state.

\subsection{The radio spectrum}

Knowing the power emitted per unit frequency in each slice of the jet (Eq. (13)) and the turn-over frequency as a function of $z$ (Eq. (15)), the spectrum of the radiation emitted by this slice can be approximated by the optically thin emission for $v>v_{\mathrm{t}}$ that is smoothly connected to the optically thick one for $v<v_{\mathrm{t}}$. Adding the contribution of each slice of the jet from $z_{0}$ to $H$, we have the total synchrotron emission in the rest frame of the jet, $P(v)$. 
For two symmetric jets streaming in opposite directions, the total flux that reaches the observer located at distance $d$ from the black hole in a direction that makes angle $\theta$ with the axis of the approaching jet is (see, e.g., Mirabel \& Rodríguez 1999)

$f(v)=\frac{P(v)}{4 \pi d^{2}}\left(\delta_{\text {apr }}^{2-s}+\delta_{\text {rec }}^{2-s}\right)$,

where $s$ is the energy spectral index $\Delta \log [P(v)] / \Delta \log (v)$. The Doppler formulae for the approaching, $\delta_{\text {apr }}$, and the receding, $\delta_{\text {rec }}$, jets are given by

$\delta_{\text {apr }}=\frac{1}{\gamma_{\|}\left(1-v_{\|} / \cos \theta\right)}$,

$\delta_{\mathrm{rec}}=\frac{1}{\gamma_{\|}\left(1+v_{\|} / \cos \theta\right)}$.

Finally, $\gamma_{\|}=1 / \sqrt{1-v_{\|}^{2} / c^{2}}$ is the bulk Lorentz factor of the jet.

For $\cos \theta \simeq 0.5$ and $v_{\|}=0.8 c$, the Doppler shifts are $\delta_{\text {apr }} \simeq$ $1, \delta_{\text {rec }} \simeq 0.4$. So, for an intermediate range of viewing angles of our mildly relativistic jet, the contribution of the receding jet to the total emitted synchrotron flux is rather small (see Eq. (18)). In principle, we should have distinguished between the photon frequency in the observer and in the comoving frame. The two frequencies scale with a Doppler factor $\delta_{\text {apr }}$. Since, $\delta_{\text {apr }} \simeq 1$ in our study, this distinction is not crucial and has been avoided.

In Fig. 3 we plot the synchrotron flux that reaches the observer, for a source that is located at distance $d=1 \mathrm{kpc}$, for different values of $\alpha$ and $B_{0}$. The rest of the parameters are kept at their reference values. Several comments are in order in view of this plot:

i) The low-frequency part of the spectrum $\left(10^{8}-10^{15} \mathrm{~Hz}\right)$ has a power-law form with slope given by Eq. (17).

ii) The low-frequency cutoff (at $\sim 10^{8} \mathrm{~Hz}$ ) is given by the value of the turn-over frequency at the outer edge of the jet at height $H$, while the high frequency cutoff (at $\sim 10^{15} \mathrm{~Hz}$ ) is close to the turn-over frequency at the base of the jet $z_{0}$. This break typically lies close to the optical region.

iii) For frequencies $\gtrsim 10^{15} \mathrm{~Hz}$, the whole volume of the jet is optically thin to synchrotron absorption and the spectrum has a power law shape with slope $-(\alpha-1) / 2$ (see Eq. (13)). The high frequency spectrum steepens further as a result of the fact that the energy distribution of the electrons extends up to $\gamma_{\max }$. Equation (13) is accurate up to frequency $v=(3 / 2) \gamma_{\max }^{2} v_{\mathrm{L}}$, where $v_{\mathrm{L}}=e B /\left(2 \pi m_{\mathrm{e}} c\right)$ is the Larmor frequency.

iv) When normalized to a distance of $1 \mathrm{kpc}$, the radio emission of black hole binaries in the low/hard state reaches fluxes of up to a few 100 mJy (see Fig. 6 in Gallo et al. 2003). Magnetic field strengths of the order of $10^{6} \mathrm{G}$ are required at the base of the jet to explain the most luminous (in the radio) sources for $3 \lesssim \alpha \lesssim 5$.

The part of the spectrum close to the high-frequency break of the flat part (close to $10^{15} \mathrm{~Hz}$ ) is rather approximate. Close to the base of the jet the characteristic radius of the jet is comparable or even larger than its height, making our analysis inaccurate for this region. Furthermore, close to the base of the jet,

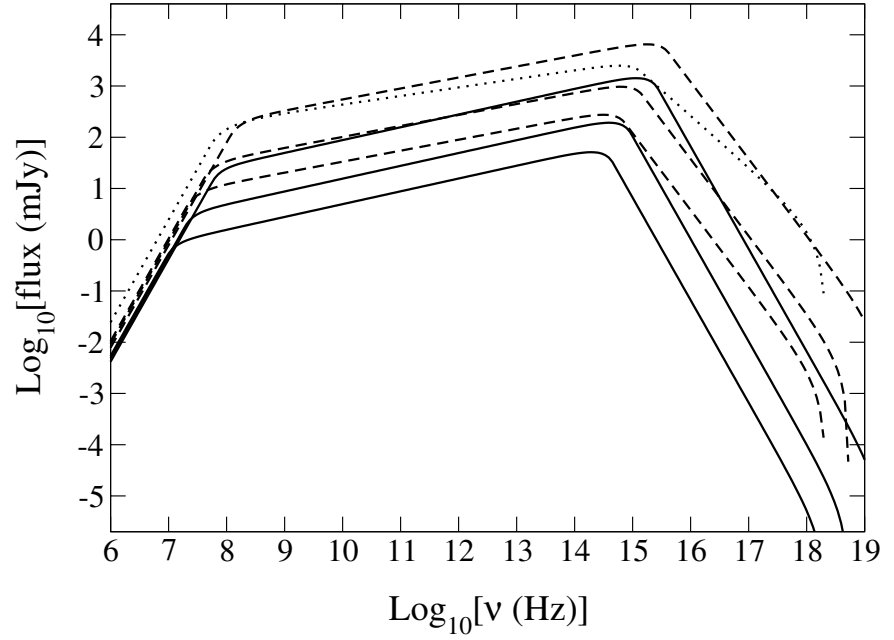

Fig. 3. The emitted synchrotron flux (in mJy) as observed at a distance of $1 \mathrm{kpc}$ from our jet is presented for different values of the index $\alpha$ and the magnetic field strength at the base of the jet $B_{0}$. The rest of the parameters are kept at their reference values (see Sect. 3). The dotted curve corresponds to $\alpha=3$ and $B_{0}=2 \times 10^{5} \mathrm{G}$. The dashed curves correspond, in order of increasing flux, to $\alpha=4$ and $B_{0}=2 \times 10^{5}, 5 \times$ $10^{5}$, and $2 \times 10^{6} \mathrm{G}$ respectively. The solid curves correspond to $\alpha=5$ and $B_{0}=2 \times 10^{5}, 5 \times 10^{5}$, and $2 \times 10^{6} \mathrm{G}$ in order of increasing flux. The spectrum in the radio is almost flat with spectral index that depends weakly on $\alpha$ (see Eq. (17)). For frequencies above $\sim 10^{15} \mathrm{~Hz}$ the whole jet is optically thin to synchrotron absorption and has slope $(1-\alpha) / 2$.

the electron scattering optical depth is high enough that it cannot be neglected. In fact, the synchrotron flux emitted close to the base of the jet may contribute significantly to the soft photon input that is Comptonized by the jet. In the next section where comparison between the spectra calculated from the model and the observational data is made, the synchrotron photons are also taken into account as a soft photon source to be Comptonized in the jet.

\section{Applying the model to XTE J1118+480 and Cygnus X-1}

The synchrotron flux, as predicted by our jet model, peaks at $\sim 10^{15} \mathrm{~Hz}$. Most of the contribution at this frequency comes from the lower region of the jet. On the other hand, synchrotron photons that are emitted in this region have a significant probability of being scattered by electrons once or more times. So, synchrotron emission must also be taken into account along with the disk emission as a soft photon source.

For the black-hole binary XTE J1118+480, simultaneous (or nearly simultaneous) observations have been conducted on multiple occasions at radio, infrared, optical, UV, EUV, and X-ray wavelengths (Hynes et al. 2000; McClintock et al. 2001b; Frontera et al. 2001), making it an ideal source to test the model in the whole electromagnetic spectrum. In Fig. 4 the most complete spectral energy distribution of XTE J1118+480 is shown (associated with the so-called "epoch 2"), where the radio data are from Fender et al. (2001; we do not include the observational point at $350 \mathrm{GHz}$ measurement which was not done simultaneously with the others) and the 


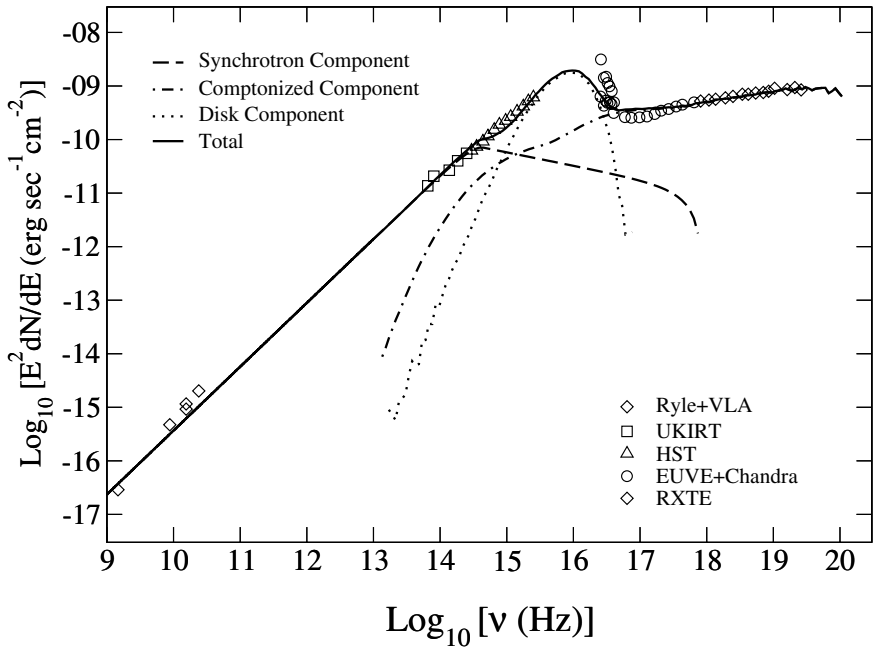

Fig. 4. The broad-band spectrum from our model for $R_{0}=70 r_{\mathrm{g}}$, $\alpha=3.5, T_{\mathrm{bb}}=10 \mathrm{eV}$, and $B_{0}=10^{5} \mathrm{G}$ with the rest of the parameters kept at their reference values (solid curve) is overplotted with the radio to X-ray spectrum of XTE J1118+480. The dashed, dashdotted and dotted curves show the synchrotron, Comptonized and disk components respectively. Notice that the EUV spectrum depends sensitively on the assumed $N_{\mathrm{H}}$ and is derived for $N_{\mathrm{H}}=1.0 \times 10^{20} \mathrm{~cm}^{-2}$ and $N_{\mathrm{H}}=1.3 \times 10^{20} \mathrm{~cm}^{-2}$ (McClintock et al. 2001b).

infrared to X-ray data from McClintock et al. (2001a). Since the EUV spectrum depends sensitively on the assumed $N_{\mathrm{H}}$ which is not well constrained but probably lies in the range $1.0-1.3 \times 10^{20} \mathrm{~cm}^{-2}$ (McClintock et al. 2001b), we choose to plot the data corrected for $N_{\mathrm{H}}=1.0 \times 10^{20} \mathrm{~cm}^{-2}$ and $N_{\mathrm{H}}=1.3 \times 10^{20} \mathrm{~cm}^{-2}$. The spectrum of XTE J1118+480 has been fitted by an accretion-jet model (Yuan et al. 2005; see also Esin et al. 2001), a synchrotron model (Markoff et al. 2001) and a thermal-Comptonization model (Frontera et al. 2001); for a comparison among different models see Chaty et al. (2003). Notice that in the ADAF and corona models the presence of a jet is needed to explain the observed emission in the infrared and radio wavelengths.

In Fig. 4, the spectrum from our model is also shown. The parameters that have been used are $R_{0}=70 r_{\mathrm{g}}, \alpha=3.5$, $T_{\mathrm{bb}}=10 \mathrm{eV}$ and $B_{0}=10^{5} \mathrm{G}$, while the rest of the parameters have been kept to their reference values. The overall agreement between the calculated and observed spectra is very good. Both disk and synchrotron photons from the jet contribute an important fraction of the soft flux to be Comptonized in the jet.

It is also clear from Fig. 4 that in this model the optically thin synchrotron emission has only a minor contribution to the $\mathrm{X}$-ray spectrum in contrast to the jet model of Markoff et al. (2001). The main reason for this discrepancy is the steeper distribution function of the emitting electron that we favor (which results in steeper optically thin synchrotron spectra) and the higher electron densities in our jet model (that result in efficient inverse Compton scattering in the jet). Recently, Heinz (2004) has shown that, adding radiative cooling to the scale invariance formalism of Heinz \& Sunyaev (2003), which is in accordance with the "fundamental plane" of black hole activity (Merloni et al. 2003), Compton scattering is favored over synchrotron as

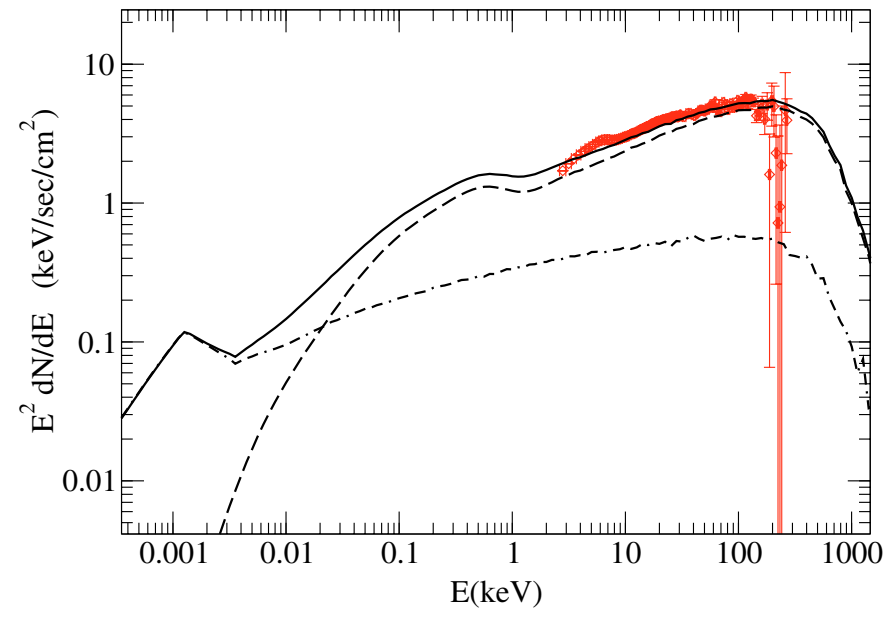

Fig. 5. The photon-number spectrum multiplied by $E^{2}$ for the reference values of our jet model (solid curve) is overplotted with the spectrum of Cygnus X-1 as observed by RXTE in December 1996. The dashed and dashed-dotted curves show the disk+Comptonized disk and synchrotron self Compton components respectively.

the emission mechanism of the hard X-rays for the majority of these sources.

Besides its broad-band spectral information, XTE J1118+ 480 has also been extensively observed in the temporal domain where a large number of interesting properties has been revealed for the lightcurves in different energy bands. For example, quasi-periodic oscillations have been observed with the same frequency in the optical, UV and X-rays (Hynes et al. 2003), and the correlation between emission at different wavelengths is clear and puzzling (Kanbach et al. 2001; Spruit \& Kanbach 2002; Hynes et al. 2003). It is interesting (though not within the scope of this work) to see whether our jet model is able to reproduce these observations.

We now proceed to apply the model to the December 1996 RXTE observations of Cygnus X-1 when the source was in the low/hard state. For the reference values of the parameters, the simulated spectra are in rather good agreement with the observed ones (see Fig. 5). On the other hand, the model underpredicts the emitted flux for $E \lesssim 30 \mathrm{keV}$.

This is the region where the reflection component, considered to come from hard X-rays that are reprocessed or reflected by the thin disk, is expected to contribute most. In this work, the reflection component has not been included in the calculation. Nevertheless, we have computed (using the Monte Carlo simulation for the reference values of the parameters) that, assuming that the disk is infinitely thin and that the jet is at an angle of 90 degrees to the disk plane, $\sim 4 \%$ of the X-ray flux hits the disk. If the disk is flared or warped (e.g. Dubus et al. 1999 ) then $\sim 8 \%$ of the X-ray flux reaches the disk (taking an "effective" $h / R=0.2$, where $h$ is the disk half-thickness and $r$ the radial distance). The fraction of X-rays that hits the disk can increase further if the jet is misaligned with respect to the outer disk (Maccarone 2002). Thus the reflection component can be rather strong and depends sensitively on the accretion-jet geometry and the velocity of the jet. More detailed modeling is 
needed to accurately compute the strength of this component as predicted by the model.

\section{Conclusion}

The hard X-ray emission in black-hole binaries is usually modeled to come from inverse Compton scattering of soft photons in a hot corona (e.g. Poutanen \& Fabian 1999) that lies above and below a thin disk (Shakura \& Sunyaev 1973) or in a twotemperature flow (Shapiro et al. 1976). Here we explore an alternative picture where the inverse Compton scattering takes place in the jet whose presence, whenever the source is in the low/hard state, is well established (Fender 2001; Stirling et al. 2001; Gallo et al. 2003).

In Paper I, it was shown that, if the density profile of the jet drops inversely proportionaly to distance, power-law spectra with photon number index 1.5-2, a cutoff at a few $100 \mathrm{keV}$ and phase lags almost independent of Fourier frequency can be derived for a wide range of the model parameters. Furthermore in Paper II, assuming that the more energetic electrons lie at small polar distances in the jet, both the hardening of the highfrequency power spectrum and the narrowing of the autocorrelation function with increasing photon energy could be reproduced, in agreement with observations of Cygnus X-1.

Here, we modified this jet model to include a steep powerlaw electron distribution (with $3 \lesssim \alpha \lesssim 5$, that is a result of fast inverse Compton - and synchrotron - cooling of the electrons) in the jet and checked that all the results derived in the X-ray region still hold. We also modeled the emission that comes from the jet at longer wavelengths in terms of synchrotron emission of the same population of electrons and found that the flat to inverted radio spectra, observed in black-hole candidates in the low/hard state, are a natural outcome of the model. Magnetic fields of the order of $10^{5}-10^{6} \mathrm{G}$ at the base of the jet are needed for the emitted power in the radio to match the typically observed one.

The flat radio spectra have been shown to extend up to about the optical wavelengths, with the base of the jet emitting mostly in this energy band. This synchrotron flux may be strong enough to contribute significantly to the soft photon input that is Comptonized in the jet. The model has been applied to the broad band spectral energy distribution of $\mathrm{J} 1118+480$ and Cygnus X-1 showing good agreement with observations.

Acknowledgements. I would like to thank Nick Kylafis for very useful discussions and comments on this manuscript. I also wish to thank the anonymous referee for comments that greatly improved and clarified the manuscript. This research has been supported by the Program "Heraklitos" of the Ministry of Education of Greece.

\section{References}

Abramowicz, M. A., Chen, X. M., Kato, S., Lasota, J. P., \& Regev, O. 1995, ApJ, 438, L37

Band, D., \& Grindlay, J. E. 1986, ApJ, 311, 595

Blandford, R. D., \& Königl, A. 1979, ApJ, 232, 34

Chaty, S., Haswell, C. A., Malzac, J., et al. 2003, MNRAS, 346, 689
Corbel, S., \& Fender, R. P. 2002, ApJ, 573, 35

Corbel, S., Fender, R. P., Tzioumis, A. K., et al. 2000, A\&A, 359, 251

Corbel, S., Nowak, M. A., Fender, R. P., Tzioumis, A. K., \& Markoff, S. 2003, A\&A, 400, 1007

Dhawan, V., Mirabel, I. F., \& Rodríguez, L. F. 2000, ApJ, 543, 373

Dubus, G., Lasota, J., Hameury, J., \& Charles, P. 1999, MNRAS, 303, 139

Esin, A. A., McClintock, J. E., \& Narayan, R. 1998, ApJ, 500, 523

Esin, A. A., McClintock, J. E., Drake, J. J., et al. 2001, ApJ, 555, 483

Falcke, H., Körding, E., \& Markoff, S. 2004, A\&A, 414, 895

Fender, R. P. 2001, MNRAS, 322, 31

Fender, R. P. 2003, in Compact Stellar X-Ray Sources, ed. W. H. D. Lewin, \& M. M. van der Klis (Cambridge University Press)

Fender, R. P., Pooley, G. G., Durouchoux, P., Tilanus, R. P. J., \& Brocksopp, C. 2000, 312, 853

Fender, R. P., Hjellming, R. M., Tilanus, R. P. J., et al. 2001, MNRAS, 322, L23

Ford, E. C., van der Klis, M., Méndez, M., van Paradijs, J., \& Kaaret, P. 1999, ApJ, 512, L31

Frontera, F., Zdziarski, A. A., Amati, L., et al. 2001, ApJ, 561, 1006

Fuchs, Y., Mirabel, I. F., Rodríguez, L. F., et al. 2003, A\&A, 409, L35

Galeev, A. A., Rosner, R., \& Vaiana, G. S. 1979, ApJ, 229, 318

Gallo, E., Fender, R. P., \& Pooley, G. G. 2003, MNRAS, 344, 60

Georganopoulos, M., Aharonian, F. A., \& Kirk, J. G. 2002, A\&A, 388, L25

Giannios, D., Kylafis, N. D., \& Psaltis, D. 2004, A\&A, 425, 163 (Paper II)

Haardt, F., \& Maraschi, L. 1993, ApJ, 413, 507

Haardt, F., Maraschi, L., \& Ghisellini, G. 1994, ApJ, 432, L95

Hannikainen, D. C., Hunstead, R. W., Campbell-Wilson, D., \& Sood, R. K. 1998, A\&A, 337, 460

Heinz, S. 2004, MNRAS, 355, 835

Heinz, S., \& Sunyaev, R. A. 2003, MNRAS, 343, 59

Hjellming, R. M., \& Han, X. 1995, in Radio properties of X-ray binaries, ed. W. H. G. Lewin, J. van Paradijs, \& E. P. J. van der Heuvel, X-ray binaries (Cambridge: Cambridge University Press), 308

Hynes, R. I., Mauche, C., Shrader, C., Cui, W., \& Chaty, S. 2000, ApJ, 539, L37

Hynes, R. I., Haswell, C. A., Cui, W., et al. 2003, MNRAS, 345, 292

Ichimaru, S. 1977, ApJ, 214, 840

Kanbach, G., Straubmeier, C., Spruit, H. C., \& Belloni, T. 2001, Nature, 414, 180

Kylafis, N. D., Giannios, D., \& Psaltis, D. 2004, in X-ray Timing 2003: Rossi and Beyond, ed. P. Kaaret, F. K. Lamb, \& J. H. Swank, American Institute of Physics, 714, 101

Levinson, A., \& Blandford, R. 1996, ApJ, 456, L29

Maccarone, T. J. 2002, MNRAS, 336, 1371

Maccarone, T. J., Coppi, P. S., \& Poutanen, J. 2000, ApJ, 537, L107

Markoff, S., \& Nowak, M. A. 2004, ApJ, 609, 972

Markoff, S., Falcke, H., \& Fender, R. 2001, A\&A, 372, L25

Markoff, S., Nowak, M. A., Corbel, S., Fender, R., \& Falcke, H. 2003, A\&A, 397, 645

Martí, J., Mirabel, I. F., Rodríguez, L. F., \& Smith, I. A. 2002, 386, 571

McClintock, J. E., \& Remillard, R. E. 2005, in Compact Stellar X-ray Sources, ed. W. H. G. Lewin, \& M. M. van der Klis (Cambridge: Cambridge University Press) [arXiv: astro-ph/0306213]

McClintock, J. E., Garcia, M. R., Caldwell, N., et al. 2001a, ApJ, 551, L147

McClintock, J. E., Haswell, C. A., Garcia, M. R., et al. 2001b, ApJ, 555,477

Merloni, A., Heinz, S., \& di Matteo, T. 2003, MNRAS, 345, 1047

Mirabel, I. F., \& Rodríguez, L. F. 1999, ARA\&A, 37, 409 
Mirabel, I. F., Rodríguez, L. F., Corbier, B., Paul, J., \& Lebrun, F. 1992, Nature, 358, 215

Mirabel, I. F., Dhawan, V., Chaty, S., et al. 1998, A\&A, 330, L9

Mitsuda, K., Inoue, H., Koyama, K., et al. 1984, PASJ, 36, 741

Miyamoto, S., Kitamoto, S., Mitsuda, K., \& Dotani, T. 1988, Nature, 336,450

Narayan, R., \& Yi, I. 1994, ApJ, 428, L13

Nowak, M. A. 1995, PASP, 107, 1207

Nowak, M. A., Vaughan, B. A., Wilms, J., Dove, J. B., \& Begelman, M. C. 1999, ApJ, 510, 874

Poutanen, J. 1998, in Theory of Black Hole Accretion Disks (Cambridge: Cambridge Univ. Press), 100

Poutanen, J., \& Fabian, A. C. 1999, MNRAS, 306, L31

Pozdnyakov, L. A., Sobol, I. M., \& Sunyaev, R. A. 1983, Astrophys. \& Space Phys. Rev., 2, 189

Rees, M. J., Phinney, E. S., Begelman, M. C., \& Blandford, R. P. 1982, Nature, 295, 17

Reig, P., Kylafis, N. D., \& Giannios, D. 2003, A\&A, 403, L15 (Paper I)

Revnivtsev, M., Gilfanov, M., \& Churazov, E. 2000, A\&A, 363, 1013

Romero, G. E., Kaufman Bernadó, M. M., \& Mirabel, F. 2002, A\&A, 393, L61
Rybicki, G. B., \& Lightman, A. P. 1979, Radiative Processes in Astrophysics (New York: Wiley)

Stern, B. E., Poutanen, J., Svensson, R., Sikora, M., \& Begelman, M. C. 1995, ApJ, 449, L13

Stirling, A. M., Spencer, R. E., de la Force, C. J., et al. 2001, MNRAS, 327,1273

Shakura, N. I., \& Sunyaev, R. A. 1973, A\&A, 24, 337

Shapiro, S. L., Lightman, A. P., \& Eardley, D. M. 1976, ApJ, 204, 187

Spruit, H. C., \& Kanbach, G. 2002, A\&A, 391, 225

Sunyaev, R. A., \& Titarchuk, L. G. 1980, A\&A, 86, 121

Tanaka, Y., \& Lewin, W. H. G. 1995, in X-ray Binaries, ed. W. H. G. Lewin, J. van Paradijs, \& E. P. j. van den Heuvel (Cambridge: Cambridge University Press), 126

Vadawale, S. V., Rao, A. R., \& Chakrabarti, S. K. 2001, A\&A, 372, 793

van der Klis, M. 1995, in The lives of neutron stars (Kluwer Academic Publishers), ed. M. A. Alpar, Ü. Kiziloglu, \& J. van Paradijs, NATO ASI Ser. C450

Yuan, F., Cui, W., \& Narayan, R. 2005, ApJ, 620, 905 